\title{
Calcium Dynamics Regulate Protective Responses and Growth of Staphylococcus aureus in Macrophages
}

https://doi.org/10.1515/bmc-2020-0021

received May 12, 2020; accepted December 24, 2020.

Abstract: Staphylococcus aureus (S. aureus) is a grampositive bacteria, which causes various fatal respiratory infections including pneumonia. The emergence of Methicillin-Resistance Staphylococcus aureus (MRSA) demands a thorough understanding of host-pathogen interactions. Here we report the role of calciumin regulating defence responses of $S$. aureus in macrophages. Regulating calcium fluxes in cells by different routes differentially governs the expression of $\mathrm{T}$ cell costimulatory molecule CD80 and Th1 promoting IL-12 receptor. Inhibiting calcium influx from extracellular medium increased expression of IFN-y and IL-10 while blocking calcium release from the intracellular stores inhibited TGF- $\beta$ levels. Blocking voltage-gated calcium channels (VGCC) inhibited the expression of multiple cytokines. While VGCC regulated the expression of apoptosis protein Bax, extracellular calcium-regulated the expression of Cytochrome-C. Similarly, VGCC regulated the expression of autophagy initiator Beclin-1. Blocking VGCC or calcium release from intracellular stores promoted phagosome-lysosome fusion, while activating VGCC inhibited phagosomelysosome fusion. Finally, calcium homeostasis regulated intracellular growth of Staphylococcus, although using different mechanisms. While blocking extracellular calcium influx seems to rely on IFN-y and IL-12R $\beta$ receptor mediated reduction in bacterial survival, blocking either

\footnotetext{
*Corresponding authors: Chaitenya Verma, Krishnamurthy Natarajan, Infectious Disease Immunology Lab, Dr. B.R. Ambedkar Center for Biomedical Research, University of Delhi, Delhi 110007, India, E-mail: chaitenya999@gmail.com; knatarajan@acbr.du.ac.in Ankush Kumar Rana, Vandana Anang, Brijendra K Tiwari, Aayushi Singh, Shakuntala Surender Kumar Saraswati, Infectious Disease Immunology Lab, Dr. B.R. Ambedkar Center for Biomedical Research, University of Delhi, Delhi 110007, India Chaitenya Verma, Department of Pathology, Wexner Medical Center, The Ohio State University, OH-43210, USA

Malini Shariff, Department of Microbiology, Vallabhbhai Patel Chest Institute, University of Delhi, Delhi 110007, India
}

intracellular calcium release or via VGCC route seem to rely on enhanced autophagy mediated reduction of intracellular bacterial survival. These results point to fine-tuning of defence responses by routes of calcium homeostasis.

Keywords: Calcium; Staphylococcus aureus; T-cell, Autophagy; Phagolysosome.

\section{Introduction}

Staphylococcus aureus (S. aureus) is a ubiquitous, grampositive bacterium. It is associated with a wide range of infections such as skin lesions (boils, sties) and localized abscess in other sites. It is commonly present on the skin and mucosal surface of the human body. They initially invade skin or mucosal barriers and then affect other organs, resulting in the development of life-threatening infections such as bacteremia, pneumonia and osteomyelitis. It plays a major role in causing hospitalacquired infections and increases disease burden. This pathogen can escape the phagolysosomal pathway $[1,2]$ and induce autophagic cell death [3] strategically that allows it to replicate and survive inside the host. S. aureus expresses several potential exotoxins. Clinically, it causes toxic shock syndrome and causes host vascular damages [4]. These toxins tamper several defence mechanisms like $\mathrm{T}$ cell activation and function, apoptosis and autophagy and also phagolysosome fusion [5-7]. Staphylococcal hemolysin A (Hla) is one of the extracellular proteins that promote pore formation and helps in the internalization of the bacterium into the host cells [8]. It alters the ion channels by changing the lipid environment and ionpermeability of the plasma membrane [9].

Calcium is one of the important ions that play an imperative role in host immune responses during infections. It facilitates cell survival through activating transcription factors, cell proliferation and development of phagolysosome fusion [10]. S. aureus infection of 
epithelial cells modulates calcium mobilization [8]. Our lab has been extensively characterizing the role of calcium homeostasis during $M$. $t b$ and BCG infections. We showed that inhibiting VGCC upon $M$. $t b$ infection induces $\mathrm{T}$ cell mediated protective immunity and significantly attenuates bacterial load in mice [11, 12]. Alternatively, activating VGCC induced suppressor responses by downmodulating oxidative burst, apoptosis, autophagy and mycobacterial survival [13]. We also demonstrated that the route of calcium entry regulated the survival of macrophages during $M . t b$ and HIV co-infection [14]. Additionally, we also showed that calcium homeostasis regulated the expression of $\mathrm{T}$ cell inhibitory molecule PD-L1 on dendritic cells during Streptococcus pneumonia infection [15].

Keeping the above, in mind in this paper, we investigated the role of calcium homeostasis in regulating defence responses to $S$. aureus infection in macrophages. Our data show that similar to mycobacteria or Streptococcus calcium homeostasis also regulated various aspects of macrophage responses to Staphylococcus aureus infection. This included the expression of $\mathrm{T}$ cell costimulatory molecules, cytokine expression, autophagy, phagolysosome fusion and survival of the pathogen.

\section{Materials and Methods}

Materials: Fluorescence-tagged antibodies against mouse CD80 (PE) (cat \# 553769) and IL-12Rß1 (PE) (cat \# 551974) were from BD Biosciences USA. Antibodies to Beclin-1 (cat \# sc-48341), ATG-5 (cat \# sc-133158) and GAPDH (cat \# sc-365062) were from Santa Cruz Biotechnologies (Santa Cruz, CA). Antibodies to LC3-I/ II (cat \# 12741S) were procured from Cell Signaling Technology (Danvers, MA, USA). ELISA kits for IFN-y and TGF- $\beta$ estimation were from e-Biosciences (USA). Calcium modulators EGTA (ethylene glycol tetra acidic; to inhibit calcium influx from extracellular medium) [16], TMB-8 (3,4,5-Trimethoxybenzoic Acid 8-(Diethylamino) octyl Ester, Hydrochloride) (to inhibit calcium release from intracellular stores) [17], BAYK8664 to (activate L-type Voltage-gated Calcium Channel, VGCC) [18] and Amlodipine (to inhibit L-type VGCC) [19], were purchased from Sigma Chemical Co. (St. Louis, MA). Lysotracker Green (Cat \# L7526) and FM4-64 (Cat \# T3166) were purchased from Molecular Probes, Life Technologies (Invitrogen, USA). A drug-sensitive clinical isolate of S. aureus was from Vallabhbhai Patel Chest Institute, University of Delhi, India.
Cell culture and stimulations: J774 murine macrophages, a kind gift from International Centre for Genetic Engineering and Biotechnology (ICGEB), New Delhi, India were used throughout the study. The cell line was maintained in Dulbecco's modified Eagle's medium (DMEM) supplemented with 10\% fetal bovine serum (FBS) at $37^{\circ} \mathrm{C}$ under $5 \% \mathrm{CO}_{2}$. Macrophages were incubated with different calcium modulators for $2 \mathrm{~h}$ followed by infection with 20 MOI $S$. aureus infection for $2 \mathrm{~h}$ and $24 \mathrm{~h}$. The concentrations of the calcium modulators used were as

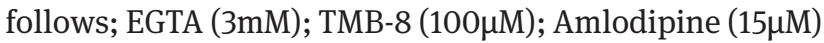
and BAYK8664 (50nM) and were based on our earlier standardizations and publications [13-15]. At the end of the incubation period, cells were processed for either flow cytometry or confocal imaging or Western blotting. The supernatants were scored for the levels of different cytokines.

Ethical approval: The conducted research is not related to either human or animals use.

Flow cytometry: Was carried out as described before [13-15, 20-22]. At the end of the incubation, cells were stained with anti-mouse CD80-PE or anti-mouse IL-12R $\beta 1$-PE antibodies for $30 \mathrm{~min}$ at room temperature. Cells were washed thoroughly twice with 1xPBS. Cells were assayed immediately using FACS Calibur (BD Biosciences). The data were plotted and analyzed using CellQuest Pro software.

\section{Confocal micr]oscopy}

For intracellular molecules: Macrophages were seeded on sterile coverslips in 12 well culture plates. Calcium modulators were used to treat the cells for $2 \mathrm{~h}$ before infection thereafter 20 MOI S. aureus for $2 \mathrm{~h}$. After incubation, cells were fixed in $4 \%$ paraformaldehyde in $1 \mathrm{x}$ PBS for 20min. Thereafter, cells were washed again with 1xPBS and permeabilized with perm-buffer containing $0.5 \% \mathrm{BSA}$ and $0.2 \%$ saponin for $15 \mathrm{~min}$; cells were washed and incubated with the indicated FITC conjugated antibody for $4 \mathrm{~h}$ at room temperature in dark. Cells were washed again and mounted with the nuclear stain DAPI. Confocal imaging was performed using Nikon C2 (Minato, Tokyo, Japan) laser scan confocal microscope with 60x magnification as described earlier [11]. Images were analysed using the NIS Elements AR software.

For Phagosome-lysosome fusion: S. aureus was labelled with $5 \mu \mathrm{g} / \mathrm{ml}$ FM4-64 dye for 2 minutes in ice, followed by thorough washes with 1xPBS, as recently described [23]. Macrophages were seeded on coverslips placed in 12 well culture plates and incubated with 
different calcium modulators for $2 \mathrm{~h}$ followed by infection with 20 MOI FM4-64 labelled S. aureus for 2h. Lysotrackergreen was used to stain lysosomes. DAPI was used to stain the nucleus. Cells were fixed with 4\% PFA and observed under Nikon C2 confocal microscope.

For Bacterial load: J774 macrophages were incubated with different calcium modulators for $2 \mathrm{~h}$ followed by infection with 20 MOI S. aureus. After $24 \mathrm{~h}$ of incubation, cells were lysed with 10\% SDS and scored for Colony Forming Units (CFU) following serial dilutions and plating onto nutrient Agar plates.

Cytokine measurement by ELISA: Macrophages were incubated with different calcium modulators for $2 \mathrm{~h}$ followed by infection with 20 MOI $S$. aureus for $24 \mathrm{~h}$. Culture supernatants were filtered with 0.2-micron filters and levels of IFN- $y$ and TGF- $\beta$ were estimated by sandwich ELISA as recommended by the manufacturer and as described before $[13,24,25]$. The standard curve was made for each cytokine to quantify the level of corresponding molecules.

Western Blotting: Immuno-blotting was carried out as described earlier [14, 23, 36]. Briefly, macrophages were incubated with different calcium modulators for $2 \mathrm{~h}$ followed by infection with 20 MOI S. aureus for $2 \mathrm{~h}$. After incubation, cells were washed with ice-cold 1x PBS and lysed using 1x RIPA buffer in the presence of 0.1M PMSF and protease inhibitors. Cytoplasmic extracts were obtained by centrifugation at 10,000g for $8 \mathrm{~min}$ at $4^{\circ} \mathrm{C}$ temperature. $30 \mu \mathrm{g}$ of cytoplasmic extract was resolved on $12 \%$ SDS-PAGE and subsequently transferred onto a nitrocellulose membrane. Blots were then probed for LC3-I/II using specific antibody followed by HRP labelled secondary antibody. Blots were later developed by chemiluminescence using ECL reagent. A parallel set of samples were run and blotted for GAPDH.

Statistical analysis: One-way ANOVA with Bonferroni's correction for multiple comparisons tests was performed to evaluate the level of significance. The P-value of less than 0.05 was considered as statistically significant. Statistical analysis and bar diagrams were plotted on GraphPad Prism version 8.4.2 for Windows, GraphPad Software, San Diego California USA.

\section{Results and Discussion}

S. aureus uses multiple strategies to avoid host immune responses. The requirement of multiple drugs makes it more imperative to understand the intricacies of hostpathogen interactions at the cellular and molecular levels.
Over the years we have been characterising the immune and defence responses mounted by dendritic cells and macrophages to infections caused by mycobacteria, HIV, Streptococcus $[14,20]$. A central molecule that regulated multiple aspects of immune responses to these infections at different levels was calcium ion concentration. We showed that not only is calcium important, but the route of calcium homeostasis also played a determinant role in regulating many processes. In this study, we explored the role of calcium in regulating some of the key defence responses to $S$. aureus infection.

\section{Calcium homeostasis regulates expression of cell surface molecules for $T$ cell activation and function}

To begin with, we first ensured that treating macrophages with calcium modulators induces changes in intracellular calcium levels. To that end macrophages loaded with calcium-sensitive dye FLUO-4-AM were treated with different calcium modulators for $1 \mathrm{~h}$ with EGTA (for blocking extracellular calcium influx), TMB8 (for blocking calcium release from intracellular stores), BAYK8644 (for activating Voltage-Gated Calcium Channels; VGCC) and Amlodipine (for blocking VGCC). Cells were incubated with these modulators for $1 \mathrm{~h}$ followed by stimulations of TLR2 (with $\mathrm{Pam}_{3} \mathrm{Csk}_{4}$ ) and TLR4 (with LPS) for $3 \mathrm{~min}$. Fluorescence from these cells was measured using a fluorescence spectrometer. As shown in Supplementary Figure 1 treatment of cells with calcium modulators alone did not appreciably modulate basal calcium levels indicating that these blockers have minimal effects. However, stimulation of TLR2 and TLR4 with specific ligands in cells treated with calcium blockers does indeed differentially modulate intracellular calcium homeostasis.

We next monitored the expression levels of costimulatory molecule CD80 and the cytokine IL-12R $\beta 1$ receptor on macrophages treated with different calcium modulators, followed by S. aureusinfection. T cellactivation occurs through the interaction of peptide presenting major histocompatibility complex (MHC) on antigenpresenting cells (APCs) (like macrophages and dendritic cells) and the T-cells receptor complex. For effective T cell activation and function, co-stimulatory molecules are critical. CD80 is an important costimulatory molecule that interacts with CD28 on the T cell surface and augments T cell activation and effector function [26, 27]. Studies also showed that CD80 promotes pro-inflammatory responses $[27,28]$. As shown in Figure 1A, infection with $S$. aureus increased CD80 expression, which was further enhanced 
A
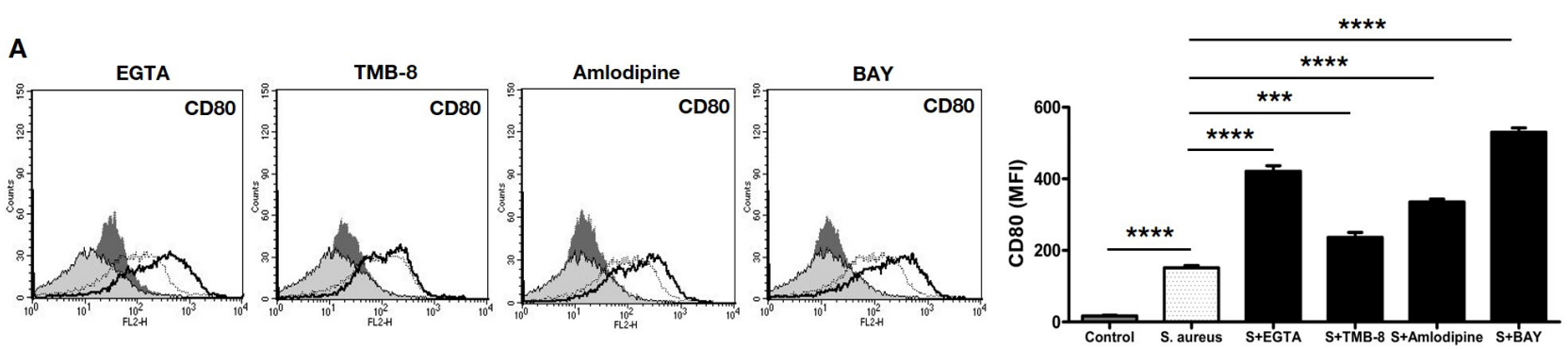

B
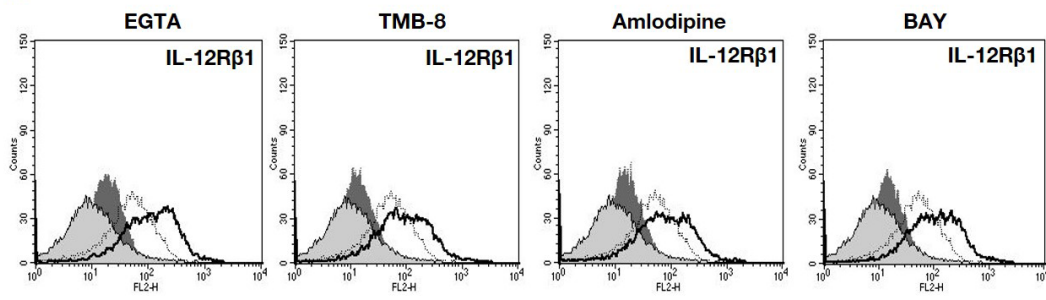

Figure 1: Calcium differentially regulates expression of T cell co-stimulatory molecules on macrophages during $S$. aureus infection.

Murine $\mathbf{7 7 4}$ macrophages were incubated with either EGTA or TMB-8 or BAYK8664 or amlodipine for 2h followed by infection with $20 \mathrm{MOI}$ S. aureus infection for 24h. Cells were stained with anti-CD80-PE (Panel A) or anti-IL-12Rß1-PE (Panel B) and analyzed by flow cytometry. Shaded histograms represent unstimulated control, dark shaded represents treatments with calcium modulators only, thin line represents infection with $S$. aureus and the thick line represents treatment with indicated calcium modulator followed by infection with $S$. aureus. Data from one of three independent experiments are shown. Bar graphs adjacent to the histograms show Mean Fluorescent Intensity (MFI) of individual groups. Controls represent unstimulated cells. Data represent the mean \pm SD of three independent experiments. S indicates S. aureus infection. For Panel A (CD80), P-value between Control and S. aureus is $p<0.0001 ;$ S. aureus vs $S$. aureus + EGTA is $p<0.0001 ; S$. aureus vs S. aureus + TMB 8 is $p=0.006 ;$ S. aureus vs $S$. aureus + Amlodipine is $p<0.0001 ;$ S. aureus vs $S$. aureus $+B A Y$ is $p<0.0001$. For Panel B (IL-12Rß1), P-value between Control vs S. aureus is $\mathrm{p}=0.0129 ;$ S. aureus vs S. aureus + EGTA is $p<0.0001 ;$ S. aureus vs $S$. aureus + TMB8 is $p<0.0001$; $S$. aureus vs $S$. aureus + Amlodipine is $p<0.0001$ and $S$. aureus vs $S$. aureus + BAYK8664 is $p<0.0001$.

upon blocking extracellular calcium influx (with EGTA) or upon activating VGCC (with BAYK8644); while blocking VGCC (with amlodipine) also increased CD80 expression over and above $S$. aureus infection. No significant role of calcium from intracellular stores was noticeable (upon blocking with TMB8). This indicated a tight regulation of CD80 expression by calcium. While a role for extracellular calcium from different sources was evident, no apparent role for intracellular calcium was observed for CD80 expression.

The profile of cytokines at the time of APC: T cell interaction plays a determinant role in regulating Th1 or Th2 responses [29-31]. These cytokines bind to their receptors on APCs as well as T cells and regulate the quality of $\mathrm{T}$ cell responses [32]. A key cytokine that mediates Th1 responses is IL-12, which binds to IL-12R and induces signals that promote APC activation as well as Th1 polarization [33, 34]. We, therefore, investigated the role of IL-12R $\beta 1$ on macrophages following calcium modulation and $S$. aureus infection. Interestingly, the expression of IL-12R $\beta 1$ was regulated by all calcium modulators (Figure 1B). Inhibiting either route of calcium homeostasis increased IL-12R $\beta 1$ expression over and above $S$. aureus infection, with maximal effects observed with inhibiting all calcium influx from extracellular medium (with EGTA). This indicated that unlike CD80 wherein calcium differentially regulated its expression, IL-12R $\beta 1$ expression was more or less dependent on calcium influx irrespective of its route of entry. It has also been shown that IL-12R present on activated NK cells or T-cells also showed higher production of IFN- $y$ and enhanced proinflammatory responses $[32,34]$ and also skewed TGF- $\beta$ and TGF- $\beta$ dependent T-cells development towards a Th1 phenotype [35]. 

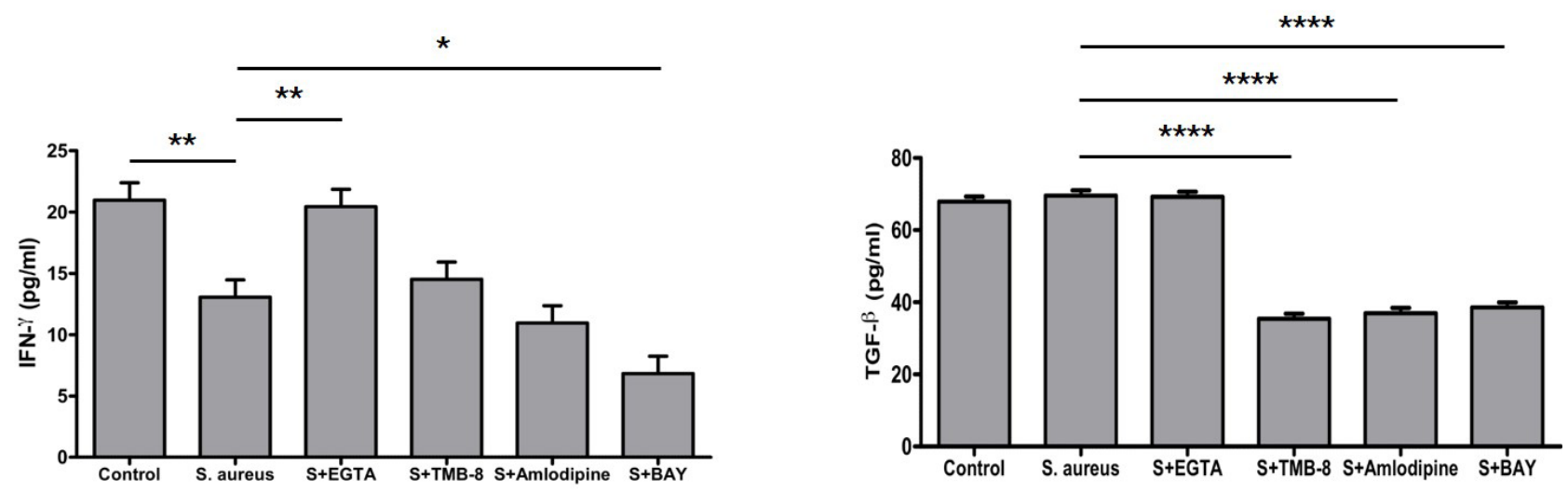

Figure 2: Calcium differentially regulates cytokine profile from macrophages during S. aureus infection. Murine J774 macrophages were incubated with either EGTA or TMB-8 or BAYK8664 or amlodipine for $2 \mathrm{~h}$ followed by infection with $20 \mathrm{MOI}$ S. aureus infection for $24 \mathrm{~h}$. Culture supernatants were scored for the levels of IFN- $\gamma$ or TGF- $\beta$. Data represent the mean \pm SD of 3 independent experiments. S indicates S. aureus infection. For IFN- $\gamma$, P-value between Control and S. aureus is $\mathrm{p}=0.0069 ;$ S. aureus vs $S$. aureus + EGTA is $\mathrm{p}=0.0099$ and $S$. aureus vs $S$. aureus + BAY is $p=0.022$. For TGF- $\beta$, P-value between-group $S$. aureus vs $S$. aureus + TMB 8 is $p<0.0001 ; S$. aureus vs $S$. aureus + Amlodipine is $p<0.0001$ and $S$. aureus vs $S$. aureus + BAYK8664is $p<0.0001$.

\section{Calcium homeostasis regulated the expression of IFN- $\gamma$ and TGF- $\beta$ from $S$. aureus infected macrophages}

Therefore, we next monitored the expression of IFN-y and TGF- $\beta$ from macrophages in the context of calcium modulation. Supplementary Figure 2 shows that calcium modulators on their own did not have any significant effects on the expression levels of either IFN-y or TGF- $\beta$. Infection of macrophages with $S$. aureus showed a decrease in IFN-y levels with no change in the expression of TGF- $\beta$ (Figure 2). Blocking VGCC significantly decreased the expression of TGF- $\beta$ without majorly affecting IFN- $\gamma$. In contrast, activating VGCC showed a different trend with a drastic decrease in IFN-y and a significant reduction in TGF- $\beta$ levels. Chelating calcium from the extracellular medium significantly increased the levels of IFN-y without affecting TGF- $\beta$ levels, while inhibiting calcium from intracellular stores did not affect IFN-y levels but did significantly decrease TGF- $\beta$ levels. Put together these results once again point towards calcium-mediated fine regulation of cytokine responses from macrophages during $S$. aureus infection.

\section{Calcium homeostasis regulates induction of autophagy from $S$. aureus infected macrophages}

In the next experiment, we investigated if the route of calcium dynamics regulates key defence responses from macrophages. Over the years it has been shown that many pathogens modulate cell survival for long-term persistence in macrophages. We earlier showed that the route of calcium entry regulated macrophage survival in an $M$. $t b$ HIV co-infection model [14]. Likewise, we also showed that mycobacteria and its proteins regulate autophagy and other post-translational modifications to their advantage $[20,36]$. Many of these processes are regulated by calcium [37-39].

We next investigated the role of calcium in regulating autophagy. S. aureus infection has been shown to modulate autophagic response for its growth [24]. This response appears necessary for replication, following destabilizing the autophagosome and leads to host cell death through apoptosis or necrosis. Studies revealed that $\alpha$-hemolysin, a pore-forming toxin secreted from $S$. aureus is a key factor responsible for the autophagic response. This response appears to be independent of Beclin-1, although it does require ATG5 [40]. Further, many studies suggest elevated cytosolic calcium stimulates autophagy and chelation of intracellular calcium inhibits autophagy [3, 41, 42]. Therefore, to investigate the role of calcium in autophagy modulation by $S$. aureus, we monitored the expression levels of key proteins in the autophagy 

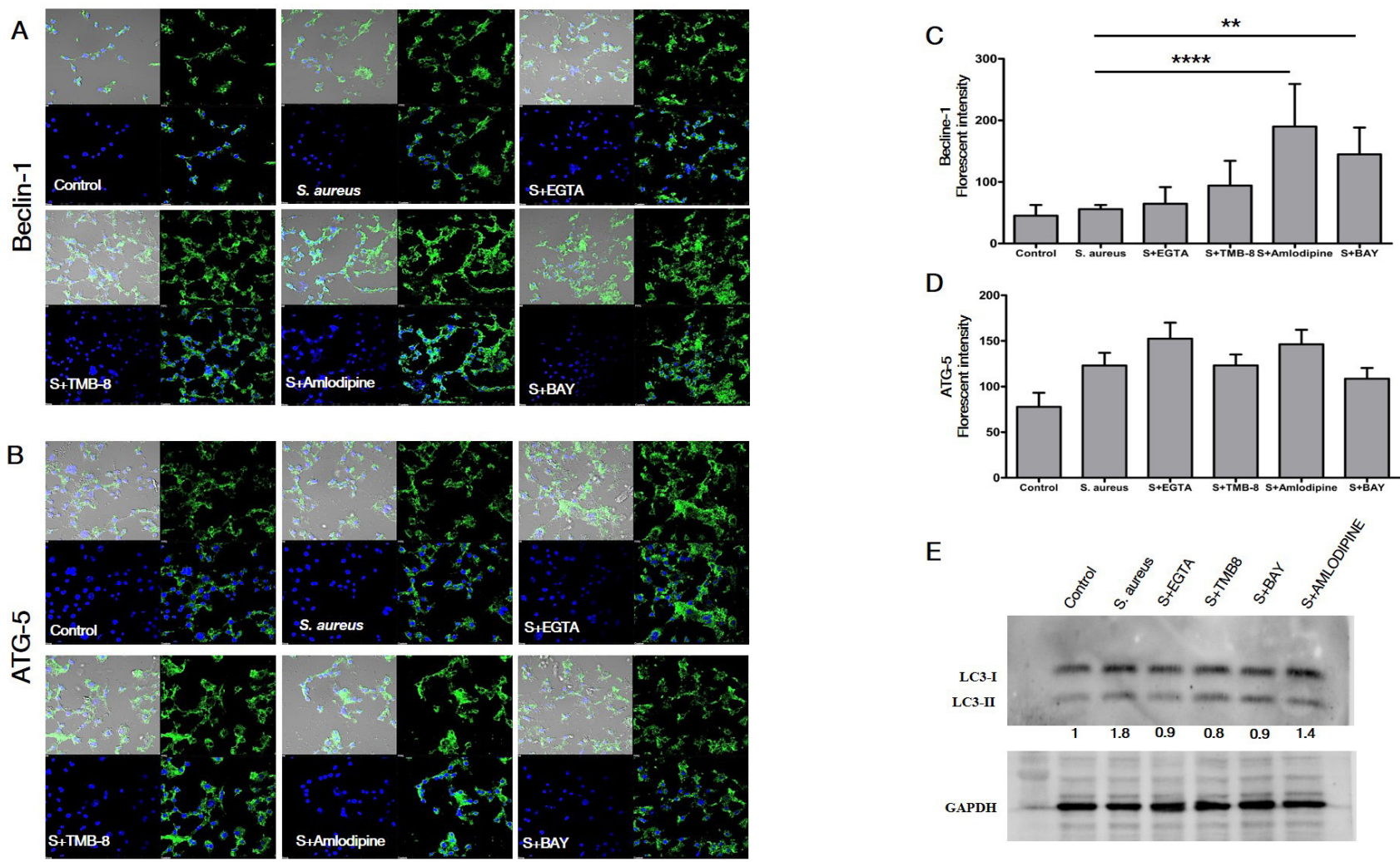

D
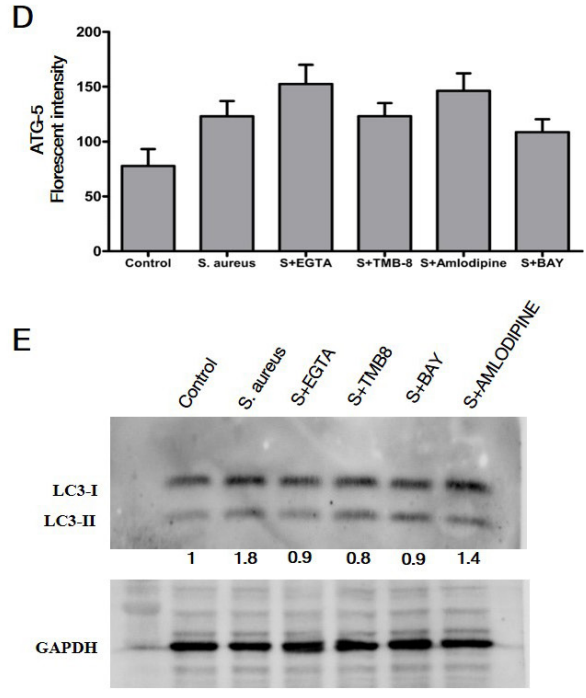

Figure 3: Calcium differentially regulates expression of autophagy pathway molecules in macrophages during $S$. aureus infection. Murine J774 macrophages were incubated with either EGTA or TMB-8 or BAYK8664 or amlodipine for $2 \mathrm{~h}$ followed by infection with 20 MOI S. aureus infection for $2 \mathrm{~h}$. Cells were processed and stained with FITC tagged antibody to Beclin-1 (Panel A) or ATG5 (Panel B). Images were captured under a confocal microscope. Confocal imaging was performed with Nikon C2 laser scan confocal microscope with 60x magnification. Data represent the mean \pm SD of 3 independent experiments. $S$ indicates $S$. aureus infection. Bar graphs adjacent to confocal images show fluorescence intensity of Beclin-1 (Panel C) and ATG-5 (Panel D), respectively. For Panel C (Beclin-1), P-value between S. aureus vs S. aureus + Amlodipine is $p<0.0001 ;$ S. aureus vs S. aureus + BAYK8664 is $p=0.0017$. For Panel E, J774 macrophages were incubated with either EGTA or TMB- 8 or BAYK8664 or amlodipine for $2 \mathrm{~h}$ followed by infection with $20 \mathrm{MOI}$ S. aureus infection for $2 \mathrm{~h}$. Cytoplasmic extracts were Western blotted for the levels of LC3-I/II. The blot for GAPDH represents loading control.

pathway, namely, Beclin-1, ATG5 and Microtubuleassociated protein 1A/1B-light chain 3 (LC3). While Beclin-1 is an initiator protein of autophagy, ATG5 feeds into the autophagosome pathway. The cytosolic form of LC3 (LC3-I) is conjugated to phosphatidylethanolamine to form LC3-phosphatidylethanolamine conjugate (LC3II), which is recruited to autophagosomal membranes [43-46].

As shown in Figure 3A blocking VGCC with amlodipine significantly increased the expression of autophagy initiator Beclin-1, while activating VGCC also increased Beclin-1 although not to the same extent as blocking VGCC. This indicated a critical threshold for VGCC function in regulating Beclin-1, thereby initiating VGCC. This indicated that blocking VGCC had a dominant role over activating VGCC in regulating Beclin-1 expression. Calcium from either extracellular medium or intracellular stores had no significant effect on Beclin-1 expression. In contrast, calcium did not have a significant effect in regulating ATG5 expression. Interestingly, however, blocking calcium release from intracellular stores, or blocking VGCC significantly increased LC3-II expression, which is the critical step in the autophagy process of engulfment of cytosolic components. Once again, maximal effects were observed upon blocking VGCC with amlodipine. The above results clearly point towards a role for calcium modulation in regulating the initiation and final step of autophagy. 

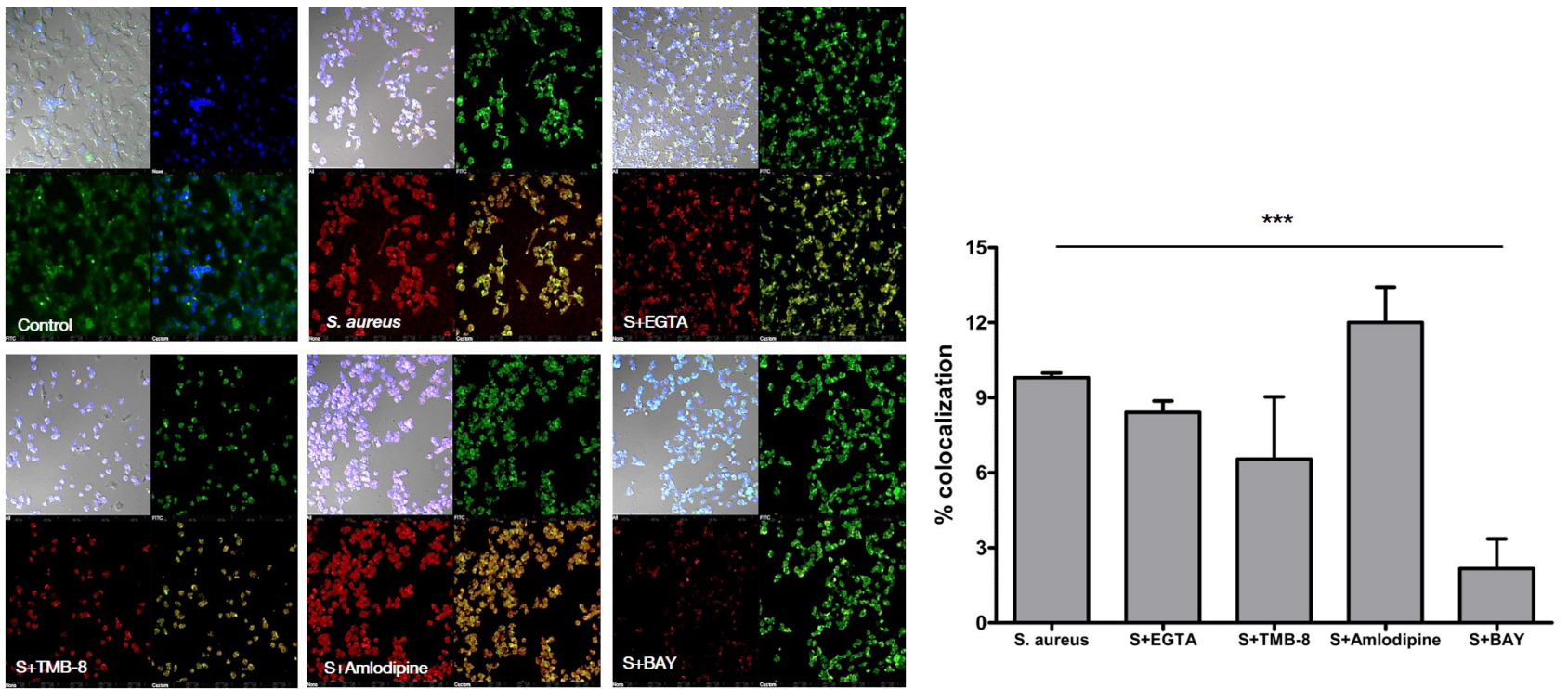

Figure 4: Calcium differentially regulates fusion of phagosomes with lysosomes in macrophages during S. aureus infection. Murine J774 macrophages were incubated with either EGTA or TMB-8 or BAYK8664 or amlodipine for $2 \mathrm{~h}$ followed by infection with FM4-64 labelled 20 $\mathrm{MOI}$ S. aureus infection for $2 \mathrm{~h}$. Following incubation cells were washed and processed and stained for Lysotracker green. Cells were fixed with $4 \%$ paraformaldehyde and mounted with DAPI. Images were analyzed using confocal microscopy. Confocal imaging was performed with Nikon C2 laser scan confocal microscope with 60x magnification. Merged images with DAPI (blue), S. aureus tagged with FM4-64 (red) and Lysotracker (green) staining are depicted. Bar graphs adjacent to confocal images show Region of Interest (ROI). Data represent mean \pm SD of three independent experiments of 10 Fields of each group. P-value between-group S. aureus vs S. aureus + BAYK8664 is p=0.0002.

\section{Calcium homeostasis regulates phagosome- lysosome fusion and bacterial survival in S. aureus infected macrophages}

Following internalization of the pathogen, the phagosome fuses with the lysosomes. This process is facilitated by calcium concentration inside of the cells [47]. Many pathogens devise multiple strategies to evade phagolysosome fusion [1]. For example, mycobacteria prevent the acidification of phagosomes, retains Rab5 on the phagosome surface and prevents the upregulation of Rab7 [48]. In addition, mycobacteria also retain Coronin-1 on phagosomes that prevent its fusion with lysosomes [49]. Trypanosoma cruzi modulates intracellular calcium to regulate phagosome-lysosome fusion [50]. We have earlier shown a critical role for VGCC in mediating phagosomelysosome fusion. Blocking VGCC promotes phagolysosome fusion while activating VGCC prevents the same [13]. In addition, we identified $M$. $t b$ protein Rv3529c that has similarity to MyD88 death domain of the TLR pathway in reducing calcium-mediated phagosome-lysosome fusion [25].

We, therefore, investigated the role of calcium modulations in regulating the fusion of phagosomes with lysosomes during S. aureus infection. As shown in Figure 4, blocking extracellular calcium influx or calcium release from intracellular stores decreased $S$. aureus induced phagosome-lysosome fusion, blocking VGCC maximally enhanced the phago-lysosome fusion. In contrast, activating VGCC dramatically inhibited the fusion of phagosomes with lysosomes. These results point towards a critical role for calcium routing towards the fusion of phagosomes with lysosomes.

Finally, weinvestigated the role of calcium in regulating the growth/survival of $S$. aureus inside macrophages. As shown in Figure 5, all calcium modulators significantly attenuated growth of $S$. aureus inside macrophages. The data suggest that the route of calcium homeostasis played a crucial role in mediating the survival of Staphylococcus in macrophages. While blocking extracellular calcium influx seems to rely on IFN-y production and IL-12R $\beta$ receptor in reducing the bacterial survival. Increased signaling from the IFN-y and IL12 receptors could mediate reduction in bacterial survival. On the other hand blocking either intracellular calcium release or via VGCC route seems to rely on enhanced autophagy mediated reduction of intracellular bacterial survival. These results indicate a complementarity of function by calcium homeostasis in regulating the survival of Staphylococcus in macrophages. 


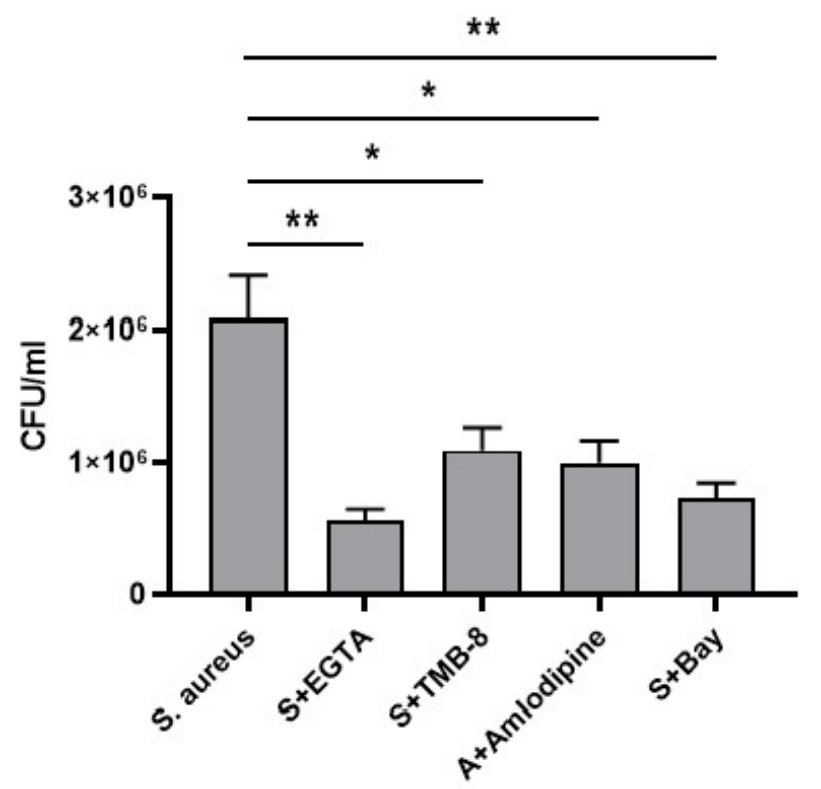

Figure 5: Calcium differentially regulates $S$. aureus survival in macrophages. Murine $\mathbf{7 7 4}$ macrophages were incubated with either EGTA or TMB- 8 or amlodipine or BAYK8664 for $2 \mathrm{~h}$ followed by $20 \mathrm{MOI}$ S. aureus infection for $24 \mathrm{~h}$. After incubation, cells were lysed with $10 \%$ SDS and serial dilutions were plated on nutrient agar plates and scored for CFUs. Data represent mean \pm SD of three independent experiments for each group. P value between group $S$. aureus vs $S$. aureus + EGTA is $p=0.0023 ; S$. aureus vs $S$. aureus + TMB- 8 is $p=0.0155$, S. aureus vs $S$. aureus + Amlodipine is $p=0.0104$ and $S$. aureus vs $S$. aureus + BAYK8664 is $p=0.0038$.

Put together, the results presented in this report have identified critical and determinant roles for routes of calcium homeostasis in regulating various aspects of $S$. aureus immunity. We show a differential requirement and fine-tuning of calcium routes in each of the parameters tested. While some routes seem to regulate $\mathrm{T}$ cell activation and function, other routes seem to regulate innate defence responses like autophagy and phagosome-lysosome fusion. A conglomeration and coordinated efforts of all these processes by calcium finally regulate the survival of S. aureus inside macrophages. A central and a net negative role of VGCC was evident wherein its blocking mediated protective responses to this pathogen.

Acknowledgements: Flow Cytometry and confocal experiment were performed at DST-FIST facility at Dr. B. R. Ambedkar Center for Biomedical Research, University of Delhi. The work was carried out using funds provided by University of Delhi and UGC-SAP-II to KN.

Conflict of interest: Authors state no conflict of interest.
Author contribution: Experimental design was formulated by $\mathrm{CV}, \mathrm{MS}$ and $\mathrm{KN}$. Experiments were performed by CV, AKR, VA, BKT, AS and SSKS. CV, MS and $\mathrm{KN}$ wrote the manuscript.

Data Availability Statement: All data generated or analyzed during this study are included in this published article and its supplementary information files.

\section{References}

1. Levin R, Grinstein S, Canton J. The life cycle of phagosomes: formation, maturation, and resolution. Immunol Rev. 2016 Sep;273(1):156-79.

2. Lacoma A, Cano V, Moranta D, Regueiro V, DomínguezVillanueva D, Laabei M, et al. Investigating intracellular persistence of Staphylococcus aureus within a murine alveolar macrophage cell line. Virulence. 2017 Nov;8(8):1761-75.

3. Bootman MD, Chehab T, Bultynck G, Parys JB, Rietdorf K. The regulation of autophagy by calcium signals: do we have a consensus? Cell Calcium. 2018 Mar;70:32-46.

4. Dinges MM, Orwin PM, Schlievert PM. Exotoxins of Staphylococcus aureus [table of contents.]. Clin Microbiol Rev. 2000 Jan;13(1):16-34.

5. Fleischer B, Schrezenmeier H. T cell stimulation by staphylococcal enterotoxins. Clonally variable response and requirement for major histocompatibility complex class II molecules on accessory or target cells. J Exp Med. 1988 May;167(5):1697-707.

6. Jonas D, Walev I, Berger T, Liebetrau M, Palmer M, Bhakdi S. Novel path to apoptosis: small transmembrane pores created by staphylococcal alpha-toxin in T lymphocytes evoke internucleosomal DNA degradation. Infect Immun. 1994 Apr;62(4):1304-12.

7. Mestre MB, Colombo MI. Staphylococcus aureus promotes autophagy by decreasing intracellular CAMP levels. Autophagy. 2012 Dec;8(12):1865-7.

8. Eichstaedt S, Gäbler K, Below S, Müller C, Kohler C, Engelmann $\mathrm{S}$, et al. Effects of Staphylococcus aureus-hemolysin A on calcium signalling in immortalized human airway epithelial cells. Cell Calcium. 2009 Feb;45(2):165-76.

9. Liang X, Ji Y. Involvement of alpha5beta1-integrin and TNFalpha in Staphylococcus aureus alpha-toxin-induced death of epithelial cells. Cell Microbiol. 2007 Jul;9(7):1809-21.

10. Berridge MJ, Lipp P, Bootman MD. The versatility and universality of calcium signalling. Nat Rev Mol Cell Biol. 2000 Oct;1(1):11-21.

11. Singh A, Anang V, Kumar Rana A, Verma C, Surender Kumar Saraswati S, Kumari P, et al. Deciphering the role of calcium homeostasis in T cells functions during mycobacterial infection. Cell Immunol. 2020 Nov;357:104198.

12. Gupta S, Salam N, Srivastava V, Singla R, Behera D, Khayyam $\mathrm{KU}$, et al. Voltage gated calcium channels negatively regulate protective immunity to Mycobacterium tuberculosis. PLoS One. 2009;4(4):e5305. 
13. Sharma D, Tiwari BK, Mehto S, Antony C, Kak G, Singh Y, et al. Suppression of Protective Responses upon Activation of L-Type Voltage Gated Calcium Channel in Macrophages during Mycobacterium bovis BCG Infection. PLoS One. 2016 Oct;11(10):e0163845.

14. Mehto S, Antony C, Khan N, Arya R, Selvakumar A, Tiwari BK, et al. Mycobacterium tuberculosis and Human Immunodeficiency Virus Type 1 Cooperatively Modulate Macrophage Apoptosis via Toll Like Receptor 2 and Calcium Homeostasis. PLoS One. 2015 Jul;10(7):e0131767.

15. Vashishta M, Khan N, Mehto S, Sehgal D, Natarajan K. Pneumococal Surface Protein A (PspA) Regulates Programmed Death Ligand 1 Expression on Dendritic Cells in a Toll-Like Receptor 2 and Calcium Dependent Manner. PLoS One. 2015 Jul;10(7):e0133601.

16. Harris RA, Hanrahan JW. Effects of EGTA on calcium signaling in airway epithelial cells. Am J Physiol. 1994 Nov;267(5 Pt 1):C1426-34

17. Doutheil J, Paschen W. Effect of 3,4,5-trimethoxybenzoic acid 8-diethylamino-octyl ester (TMB-8) on neuronal calcium homeostasis, protein synthesis, and energy metabolism. Pharmacol Toxicol. 1999 Aug;85(2):85-91.

18. Thomas G, Chung M, Cohen CJ. A dihydropyridine (Bay k 8644) that enhances calcium currents in guinea pig and calf myocardial cells. A new type of positive inotropic agent. Circ Res. 1985 Jan;56(1):87-96.

19. Lee S. Pharmacological Inhibition of Voltage-gated $\mathrm{Ca}(2+)$ Channels for Chronic Pain Relief. Curr Neuropharmacol. 2013 Dec;11(6):606-20.

20. Singhal J, Agrawal N, Vashishta M, Priya NG, Tiwari BK, Singh $\mathrm{Y}$, et al. Suppression of dendritic cell-mediated responses by genes in calcium and cysteine protease pathways during Mycobacterium tuberculosis infection. J Biol Chem. 2012 Mar;287(14):11108-21.

21. Selvakumar A, Antony C, Singhal J, Tiwari BK, Singh Y, Natarajan K. Reciprocal regulation of reactive oxygen species and phospho-CREB regulates voltage gated calcium channel expression during Mycobacterium tuberculosis infection. PLoS One. 2014 May;9(5):e96427.

22. Antony C, Mehto S, Tiwari BK, Singh Y, Natarajan K. Regulation of L-type Voltage Gated Calcium Channel CACNA1S in Macrophages upon Mycobacterium tuberculosis Infection. PLoS One. 2015 Apr;10(4):e0124263.

23. Bandyopadhyay U, Chadha A, Gupta P, Tiwari B, Bhattacharyya K, Popli S, et al. Suppression of Toll-like receptor 2-mediated proinflammatory responses by Mycobacterium tuberculosis protein Rv3529c. J Leukoc Biol. 2017 Nov;102(5):1249-59.

24. Korea CG, Balsamo G, Pezzicoli A, Merakou C, Tavarini $\mathrm{S}$, Bagnoli F, et al. Staphylococcal Esx proteins modulate apoptosis and release of intracellular Staphylococcus aureus during infection in epithelial cells. Infect Immun. 2014 Oct;82(10):4144-53.

25. Gupta D, Sharma S, Singhal J, Satsangi AT, Antony C, Natarajan K. Suppression of TLR2-induced IL-12, reactive oxygen species, and inducible nitric oxide synthase expression by Mycobacterium tuberculosis antigens expressed inside macrophages during the course of infection. J Immunol. 2010 May;184(10):5444-55.
26. Yao S, Zhu Y, Chen L. Advances in targeting cell surface signalling molecules for immune modulation. Nat Rev Drug Discov. 2013 Feb;12(2):130-46.

27. Chen L, Flies DB. Molecular mechanisms of T cell co-stimulation and co-inhibition. Nat Rev Immunol. 2013 Apr;13(4):227-42.

28. Parker D. CD80/CD86 signaling contributes to the proinflammatory response of Staphylococcus aureus in the airway. Cytokine. 2018 Jul;107:130-6.

29. Nolan A, Kobayashi H, Naveed B, Kelly A, Hoshino Y, Hoshino $S$, et al. Differential role for CD80 and CD86 in the regulation of the innate immune response in murine polymicrobial sepsis. PLoS One. 2009 Aug;4(8):e6600.

30. León B, Ballesteros-Tato A, Lund FE. Dendritic cells and B cells: unexpected partners in Th2 development. J Immunol. 2014 Aug;193(4):1531-7.

31. Kaiko GE, Horvat JC, Beagley KW, Hansbro PM. Immunological decision-making: how does the immune system decide to mount a helper T-cell response? Immunology. 2008 Mar;123(3):326-38.

32. Desai BB, Quinn PM, Wolitzky AG, Mongini PK, Chizzonite R, Gately MK. IL-12 receptor. II. Distribution and regulation of receptor expression. J Immunol. 1992 May;148(10):3125-32.

33. Murphy EE, Terres G, Macatonia SE, Hsieh CS, Mattson J, Lanier L, et al. B7 and interleukin 12 cooperate for proliferation and interferon gamma production by mouse T helper clones that are unresponsive to B7 costimulation. J Exp Med. 1994 Jul;180(1):223-31.

34. Igarashi O, Yamane H, Imajoh-Ohmi S, Nariuchi H. IL-12 receptor (IL-12R) expression and accumulation of IL-12R beta 1 and IL-12R beta 2 mRNAs in CD4+ T cells by costimulation with B7-2 molecules. J Immunol. 1998 Feb;160(4):1638-46.

35. Prochazkova J, Pokorna K, Holan V. IL-12 inhibits the TGF$\beta$-dependent T cell developmental programs and skews the TGF- $\beta$-induced differentiation into a Th1-like direction. Immunobiology. 2012 Jan;217(1):74-82.

36. Chadha A, Mehto S, Selvakumar A, Vashishta M, Kamble SS, Popli S, et al. Suppressive role of neddylation in dendritic cells during Mycobacterium tuberculosis infection. Tuberculosis (Edinb). 2015 Sep;95(5):599-607.

37. Kerr JF, Wyllie AH, Currie AR. Apoptosis: a basic biological phenomenon with wide-ranging implications in tissue kinetics. Br J Cancer. 1972 Aug;26(4):239-57.

38. Green DR. Overview: apoptotic signaling pathways in the immune system. Immunol Rev. 2003 Jun;193(1):5-9.

39. Pinton P, Giorgi C, Siviero R, Zecchini E, Rizzuto R. Calcium and apoptosis: ER-mitochondria Ca2+ transfer in the control of apoptosis. Oncogene. 2008 Oct;27(50):6407-18.

40. Mestre MB, Colombo MI. CAMP and EPAC are key players in the regulation of the signal transduction pathway involved in the $\alpha$-hemolysin autophagic response. PLoS Pathog. 2012;8(5):e1002664.

41. Sakaki K, Wu J, Kaufman RJ. Protein kinase Ctheta is required for autophagy in response to stress in the endoplasmic reticulum. J Biol Chem. 2008 May;283(22):15370-80.

42. Pfisterer SG, Mauthe M, Codogno P, Proikas-Cezanne T. Ca2+/ calmodulin-dependent kinase (CaMK) signaling via CaMKI and AMP-activated protein kinase contributes to the regulation of WIPI-1 at the onset of autophagy. Mol Pharmacol. 2011 Dec;80(6):1066-75. 
43. Tanida I, Ueno T, Kominami E. LC3 and Autophagy. Methods Mol Biol. 2008;445:77-88.

44. Marquez RT, Xu L. Bcl-2:Beclin 1 complex: multiple, mechanisms regulating autophagy/apoptosis toggle switch. Am J Cancer Res. 2012;2(2):214-21.

45. Hurley JH, Schulman BA. Atomistic autophagy: the structures of cellular self-digestion. Cell. 2014 Apr;157(2):300-11.

46. Wu H, Che X, Zheng Q, Wu A, Pan K, Shao A, et al. Caspases: a molecular switch node in the crosstalk between autophagy and apoptosis. Int J Biol Sci. 2014 Sep;10(9):1072-83.

47. Kim E, Enelow RI, Sullivan GW, Mandell GL. Regional and generalized changes in cytosolic free calcium in monocytes during phagocytosis. Infect Immun. 1992 Mar;60(3):1244-8.

48. Kelley VA, Schorey JS. Mycobacterium's arrest of phagosome maturation in macrophages requires Rab5 activity and accessibility to iron. Mol Biol Cell. 2003 Aug;14(8):3366-77.

49. Jayachandran R, Sundaramurthy V, Combaluzier B, Mueller $\mathrm{P}$, Korf $\mathrm{H}$, Huygen $\mathrm{K}$, et al. Survival of mycobacteria in macrophages is mediated by coronin 1-dependent activation of calcineurin. Cell. 2007 Jul;130(1):37-50.

50. Steinberg TH, Newman AS, Swanson JA, Silverstein SC. Macrophages possess probenecid-inhibitable organic anion transporters that remove fluorescent dyes from the cytoplasmic matrix. J Cell Biol. 1987 Dec;105(6 Pt 1):2695-702. 